\title{
On the Vertical Uniformity of an ITER-like Large Beam
}

\author{
Federica Bonomo ${ }^{\mathrm{a}^{*}}$, Isabella Mario ${ }^{\mathrm{a}}$, Dirk Wünderlich ${ }^{\mathrm{a}}$, Ursel Fantz $^{\mathrm{a}}$ \\ ${ }^{a}$ Max-Planck-Institut für Plasmaphysik, Boltzmannstr. 2, 85748 Garching, Germany
}

\begin{abstract}
A beam uniformity higher than $90 \%$ is a crucial requirement for the ITER neutral beam injection (NBI) system, together with very low beam core divergence $(<7 \mathrm{mrad})$, to maximize the beam transmission into the tokamak and to optimize the release of energy to the fusion particles.

The ELISE test facility, equipped with an ITER-like multi-aperture 3-grid system, produces large ITER-like beams, with half the size in vertical direction. Due to the distance of the beam diagnostics from the grids, the overlapping of the beamlets is large, and information on divergence and uniformity can be retrieved in terms of beamlet groups or beam segments. In general, good symmetry in accelerated current density between beam segments is achieved. The vertical beam uniformity is determined by caesium distribution and by plasma parameters at the plasma grid, which are inhomogeneous due to the interplay of filter field and bias potential. In this work, the role of filter field and bias potential on the vertical beam uniformity (in intensity and divergence) between the two beam segments is identified: the filter field mainly affects the extracted current density and its distribution within and between the segments; the bias potential can be used to tune the beam divergence, differently between the beam segments.
\end{abstract}

Keywords: Neutral Beam Injection, ELISE, negative ion source, beam diagnostics, beam homogeneity, ITER NBI

\section{The ELISE test facility towards the ITER NBI}

Two Neutral Beam Injection (NBI) lines will provide $33 \mathrm{MW}$ of total power to the ITER plasma, for heating and current drive [1]. The neutrals will be generated as negative ions and neutralized in a gas neutralizer before reaching the Tokamak. The negative ions (hydrogen or deuterium) will be produced in a caesiated RF-driven ion source, extracted and accelerated by a multi-grid multiaperture accelerator to $1 \mathrm{MV}$ for pulses up to 1 hour (deuterium); in hydrogen, $870 \mathrm{kV}$ are needed up to $1000 \mathrm{~s}$. Strict requirements are on the source filling pressure $(<0.3 \mathrm{~Pa})$ to minimize the transmission losses and the power loads on the beam line components [2].

One of the challenges of this system is to combine, for a large ion source area (almost $1 \mathrm{~m} \times 2 \mathrm{~m}$ ), high extracted negative ion current density $\left(329 \mathrm{~A} / \mathrm{m}^{2}\right.$ for hydrogen, $286 \mathrm{~A} / \mathrm{m}^{2}$ for deuterium) with a ratio of coextracted electrons to extracted ions smaller than 1 at a filling pressure of $0.3 \mathrm{~Pa}$ maximum. This has to be coupled with very strict requirements also on the beam properties: at least $85 \%$ of the power is foreseen to be carried by the 1280 beamlets, with a divergence smaller than $7 \mathrm{mrad}$ and a high uniformity (better than $90 \%$ ) [2].

In order to succeed in achieving the ITER NBI parameters, several smaller test facilities have been realized to test the feasibility and reliability of RF-driven negative ion sources. The full-size scale $100 \mathrm{keV}$ ITER NBI source SPIDER [3] is in operation at Consorzio RFX (Padua) since July 2018; the $1 \mathrm{MV}$ full-line testbed (MITICA) is still under construction [4].

In the roadmap towards the ITER NBI, the ELISE test facility [5] is the size scale source between the RFdriven prototype source [6] and the full-scale ITER NBI source, having $1 / 2$ the vertical size of the ITER NBI source, but same width. In ELISE, the same mechanism of plasma generation based on inductive RF coupling is used as for the ITER NBI source. Plasma is generated in four drivers (instead of eight), which can operate in steady state. The negative ions are mainly produced by surface conversion of neutrals on a low work function caesiated surface [7]. Caesium is evaporated in the source by two ovens [8].

ELISE is equipped with an ITER-like multi-aperture 3-grid system. The chamfered plasma-facing grid (PG), similar to the ITER NBI one, is combined with an extractor (extraction grid, EG, equipped with permanent magnets to dump the electrons before being fully accelerated) and an acceleration system (grounded grid, GG). The grid design has been adapted for a maximum potential of $60 \mathrm{kV}$. Unlike the ITER NBI grids, all the grids in ELISE are flat, not inclined, and no steering is foreseen for the beamlets. In ELISE, the beam is extracted and accelerated only in pulse mode ( $9.5 \mathrm{~s}$ every $150 \mathrm{~s}$; each beam phase is called beam blip).

The circular aperture arrangement is similar to the ITER NBI one: four groups of $16 \times 5$ apertures each, are gathered in two grid segments (instead of four as for the ITER NBI), one positioned above the other (beam segments). The whole beam results from the overlapping of 640 beamlets (1280 for the ITER NBI). Thus the ITER-relevance of the large beams of ELISE: it is possible to gather experience and knowledge on the operation of large ITER-like beams as well as to investigate beam optics properties, to improve beam diagnostic evaluation and to investigate and improve beam uniformity, though the grid design and the limited grid potentials do not allow for achieving the ITERrelevant small divergences of $7 \mathrm{mrad}$. The beam divergence foreseen for ELISE at ITER relevant current densities, with $10 \mathrm{kV}$ of extraction potential plus $50 \mathrm{kV}$ of acceleration potential, is of $\approx 20 \mathrm{mrad}$ for $\mathrm{H}^{-}$and 


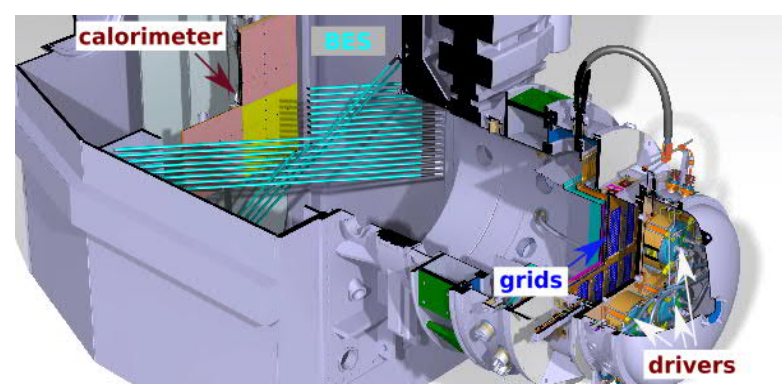

Fig. 1. Overview of the ELISE test facility and of the beam diagnostics (BES and diagnostic calorimeter).

$\approx 14$ mrad for $\mathrm{D}^{-}$[9]. Experimentally, larger divergences are measured because of the overlapping of beamlets deflected horizontally in a "zig-zag" pattern due to the magnets embedded in the EG [10]. An overall view of the ELISE source and beam diagnostics is in Figure 1.

As foreseen for the ITER NBI, on the plasma-side of the ELISE PG, a horizontal magnetic field is created by a current flowing vertically through the PG ( $I_{P G}$ of $1 \mathrm{kA} \approx 1 \mathrm{mT}$ in the source center, $2 \mathrm{~cm}$ upstream the PG [11]). The goal of the filter field (FF) is to cool down the electrons to about $1 \mathrm{eV}$ (i.e. to minimize the destruction process rate of the negative ions due to collisions with energetic electrons) and to limit the amount of co-extracted electrons. The interplay of the magnetic field with electrical fields is responsible for a vertical drift of the plasma in front of the PG (upward directed for the experiments presented in this work). Downstream of the PG, the $I_{P G}$ field causes a vertical deflection of the beam, in the opposite direction with respect to the plasma drift. To increase the field strength and modify the field topology, permanent magnets are added externally [12]; in this work, the magnets add $\approx 0.4 \mathrm{mT}$ in front of the PG to the $I_{P G}$ field.

To further decrease the amount of co-extracted electrons, the PG is positively biased with respect to the source wall, which is extended close to the aperture area by a plate (bias plate). To avoid current overshoots, the biasing is controlled in current $\left(I_{\text {bias }}\right)$; the voltage $U_{\text {bias }}$ is anyway measured. To enlarge the positively biased surface, so-called potential rods have been added vertically between the beamlet groups, upstream side of the bias plate [13]; they are kept at PG potential.

\subsection{Beam diagnostics in ELISE}

The total extracted negative ion current $\left(I_{e x}\right)$ is electrically measured as the sum of all the charged particles (negative ions) exiting the EG and impinging anywhere downstream the EG. It is used to determine the extracted negative ion current density $j_{e x}$ by dividing $I_{e x}$ with the total extraction area $\left(\approx 0.1 \mathrm{~m}^{2}\right)$. This definition neglects negative ions stripped in the PG-EG gap if the stripped electrons hit the EG. No direct measurements of the stripped electrons are possible. Detailed calculations of the stripping losses for ELISE are ongoing work; the total amount of striping losses in the EG-PG gap is expected not to exceed the $10 \%$, which is also the total amount of stripping losses foreseen for the ITER NBI system in the PG-EG gap. The current flowing in the EG is measured and mainly attributed to the co-extracted electrons $\left(I_{e l}\right)$, though stripped electrons and negative ions contributes as well. The current flowing in the GG (stripped electrons and negative ions) is measured as well. The currents in the EG and GG are measured separately in the two halves of the grids.

The total accelerated negative ion current $\left(I_{a c c}\right)$ is measured at the diagnostic calorimeter [14], located $3.5 \mathrm{~m}$ downstream of the GG (see Figure 1). $I_{a c c}$ is given via Infra-Red (IR) analysis of the temperature of the blackened-surface of the calorimeter. An IR camera looking at the calorimeter surface provides a 2D map of the temperature increase due to the power deposited. By assuming all the particles having the full acceleration energy, the total accelerated current is calculated. The spatial resolution of the $2 \mathrm{D}$ temperature map is $4 \mathrm{~cm} \times 4 \mathrm{~cm} \quad(1.2 \mathrm{~m} \times 1.2 \mathrm{~m}$ in total $) . \quad$ A $2 \mathrm{D}$ fitting routine [15] permits to retrieve the deposited power associated to each of the eight beamlet groups; by summing up the current associated to the beamlet groups belonging to the same grid segment, the accelerated current from the top and bottom grid segments can be used to estimate the beam vertical symmetry. The overlapping of the beamlets and of the beamlet groups at the calorimeter depends on the beamlet divergence and uniformity. A sensitivity study of the fitting method has shown the capability to successfully retrieve information on the vertical position (beam deflection) and size (horizontally and vertically) of the single beamlet groups, even when the overlap is so large that they are not separated in the 2D map. No direct access to the properties of the single beamlets is possible, due to the large distance of the diagnostic from the grids and its spatial resolution.

The Beam Emission spectroscopy (BES) diagnostic [15] is complementary to IR analysis, and helps in the beam property characterization. BES is based on the analysis of $\mathrm{H}_{\alpha}$ Doppler-shifted peaks originating from the interaction between fast beam particles and the background gas. The half e-folding width of the Doppler-shifted $\mathrm{H}_{\alpha}$ peak is used to estimate the beam divergence [15]. Two sets of lines of sight (LOS) - one vertical (16 LOS) and one horizontal (4 LOS) - intersect the beam at $2.7 \mathrm{~m}$ from GG with a tilted angle of $50 \mathrm{deg}$ with respect to the beam direction (see Figure 1). BES is a non-invasive measurement and the beam intensity and divergence are averaged along the lines of sight. The horizontal zig-zag deflection of the ions, alternated in direction row-by-row, broadens the resulting Doppler-shifted peaks (i.e. resulting in an apparently higher divergence) due to the beamlet mixing and large intersection of the LOS with the beam. The Doppler-shifted peak is further broadened because the light collected along the BES LOS represents the average of different strongly overlapping beamlets, which can additionally have significantly different properties [16]. Divergence estimation by BES is therefore a sort of "global" whole beam divergence, which is larger than the single beamlet divergence [10]. 
Anyway, the whole beam divergence evolves proportionally to the single beamlet divergence, thus giving indication on the divergence evolution with source and operational parameters. In general, the divergence estimated from the vertical LOS is smaller than that from the horizontal ones, because of the horizontal zig-zag effect.

Both IR analysis and BES give global and averaged information on the beam properties. To characterize the beam optic properties, perveance $\left(P=I_{e x} / U_{e x t}^{1.5} ; U_{e x t}\right.$ is the potential between EG and PG) [17] is usually used; beam divergence and perveance are linked together [18]. Divergence is also defined by the combination of the two electrostatic lenses in the two gaps, i.e. the ratio of the potentials for the two gaps, $U_{e x t}$ and $U_{a c c}$; for ELISE, the optimum ratio minimizing the divergence for a defined perveance is found to be between 5 and 6 , both in experiments and calculations [9].

The relation between the negative ion extracted current and divergence through the perveance is valid only in the case of single beamlets and for one gap (PGEG). Therefore, in a multi-aperture large beam, it holds only in case of a perfectly uniform beam (i.e. all the beamlets carry the same amount of current). In reality, the total extracted negative ion current can be given by different combinations of current density distributions among the apertures. Consequently, the relation of the total extracted negative ion current with divergence is not well defined. A "global" perveance using the total extracted negative ion current is used to investigate the beam in-homogeneities by combining the information of BES and IR. In general, the normalized perveance is considered: perveance is normalized to the maximum extractable current possible for the Child-Langmuir law [19],[20]. At ELISE, the optimum normalized perveance is typically found between $0.25-0.3$.

\section{Vertical beam symmetry and uniformity}

\subsection{General behavior of a uniform beam}

Plasma (and therefore beam) in-homogeneities are expected to be dominant in the vertical direction, mostly due to the vertical plasma drift in front of the PG caused by the interplay of the horizontal filter field with electric fields. Since there is no access to single beamlet properties, the combination of both segment symmetry and uniformity can be used to assess the beam homogeneity.

Vertical beam symmetry is linked to the extracted negative ion current, regardless of its distribution. It is evaluated as the ratio between the accelerated current from the top grid segment $\left(I_{a c c}{ }^{\text {top }}\right)$ and the bottom one $\left(I_{a c c}{ }^{b o t}\right)$; in general at ELISE is regularly in the range $I_{a c c}{ }^{t o p} / I_{a c c}{ }^{b o t}=0.9-1.1$; it can be improved by a separate tuning of the RF generators [21]

Vertical beam uniformity among the beam segments is difficult to define, since it would imply to have access to the current density distribution within the beamlet groups. This can be available (at least partially) only by

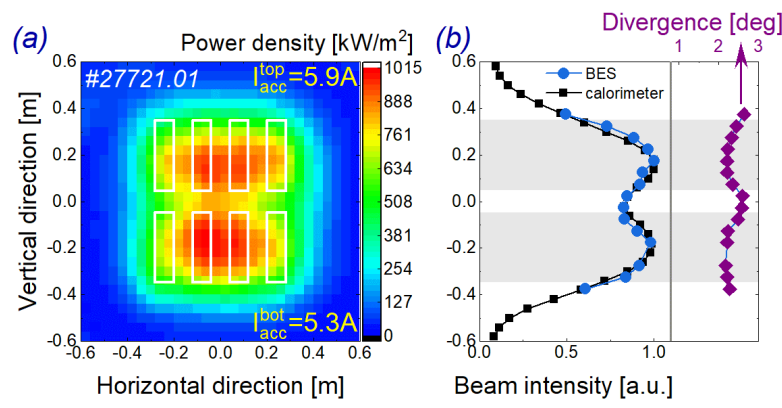

Fig. 2. (a) 2D power density map at the calorimeter and (b) the corresponding vertical profile (squares)together with the beam profile from BES (circles); no $I_{P G}$ current applied. Vertical profile of the divergence (from BES) is shown in panel (b) with diamonds. Parameters: hydrogen, $0.4 \mathrm{~Pa}$, $j_{e x}=150 \mathrm{~A} / \mathrm{m}^{2}, \quad U_{\text {bias }}=7.4 \mathrm{~V}$ (control in $I_{\text {bias }}=0 \mathrm{~A}$ ), $U_{H V}=9.2 \mathrm{kV}+33 \mathrm{kV}, F F=0.4 \mathrm{mT}$.

combining the information of the vertical divergence profile with the power density map at the calorimeter. Only in case of a perfectly uniform beam (i.e. all the beamlets carry the same current and have the same divergence), a perfectly "flat" divergence profile in correspondence of the beam segment would be detected from BES, while eight rectangular beamlet groups, well detached one from the others, would be visible in the calorimeter. Any deviation from this case has to be attributed to inhomogeneities within or among the beamlet groups.

Beam uniformity is related to a uniform work function at the PG surface and uniform plasma parameters in front of the extractor. However, plasma drifts create inhomogeneities in the plasma parameters which, in turn, affect the Cs redistribution. An example of beam with negligible plasma drifts $\left(I_{P G}=0 \mathrm{kA}\right.$, with external magnets) but with a non-uniform Cs distribution on the PF surface (determined by the Cs conditioning which is done in presence of filter field, i.e. of plasma drifts) is shown in Figure 2. 2D map of the power density at the calorimeter is displayed in panel (a); the rectangles represent the geometrical projection of the beamlet groups at the calorimeter. The vertical profile of the power density (horizontally averaged) is displayed in panel (b), in comparison with the beam intensity profile as obtained from BES. The grey areas correspond to the projections of the beamlet groups. Both the vertical intensity profiles are an average of the beam properties along the horizontal direction, and look very similar. The beam optic for this pulse is not at the optimum, so that the beam divergence (around 2-2.2 deg in correspondence of the projection of the beamlet groups) is not minimized for the voltages and the current density. As a consequence of the high divergence, the beamlet groups are indistinguishable inside the beam segments; the two beam segments are instead well detached, carrying each almost the same amount of accelerated current (symmetry $I_{a c c}{ }^{t o p} / I_{a c c}{ }^{b o t}=1.1$ ) but with different shapes. The top segment looks vertically narrower (also in the divergence profile) than the bottom one, which has a flatter divergence profile. The differences observed are 
addressed to a non uniformly caesiated PG surface, suggesting then for the necessity of an improved Cs evaporation method for ELISE.

\subsection{Influence of the horizontal filter field}

The vertical plasma drift in front of the PG depends on the strength of the horizontal component of the magnetic field, and this affects the plasma properties and potentials in front of the apertures, differently between the different segments [22].

In Figure 3, the effect on the beam symmetry and uniformity by varying the filter field strength is shown, together with the impact on the currents. The trend of the extracted and accelerated negative ion currents as well as for the co-extracted electrons are shown in (a); $I_{a c c}$ and $I_{e l}$ are displayed for the two grid segments with the upward and downward triangles. The vertical (horizontally averaged) IR profiles (b) and the vertical divergence profiles from BES (c) are displayed for different values of the $I_{P G}$ current, during hydrogen operation at the constant RF power of $48 \mathrm{~kW} /$ driver. The grey areas correspond to the position of the beamlet groups. Several observations can be made.

- The total extracted negative ion current $I_{e x}$ is slightly varying with the filter field while the co-extracted electrons are strongly and monotonically decreasing with $I_{P G}$. The optimum for $I_{e x}$ is at a reduced amount of electron current but still at a small filter field. The $I_{a c c}$ trend for both segments follows the $I_{e x}$ trend.

- The downwarded beam deflection is visible in panel (b): the beam intensity minimum in the vertical IR profiles is some centimeters below the geometrical grid center, corresponding to the position of $0.0 \mathrm{~m}$.

- The top/bottom beam symmetry stays almost constant by varying the filter field: $I_{a c c}{ }^{t o p} / I_{a c c}{ }^{b o t} \approx 1.1$, slightly better at the highest field (1.06) than at the lower one (1.17). However, the vertical profiles of beam intensity and divergence are changing a lot within the beamlet groups. In particular, the two beam segments react differently at the field increase: the bottom one is highly depressed in the vertical intensity at the highest values (more than $2 \mathrm{kA}$ ), while becoming larger (i.e. higher divergence). The top segment, instead, is not changing as much as the bottom one in the vertical intensity profile, but monotonically decreasing in divergence.

- At low $I_{P G}(0.45 \mathrm{kA}$, about $0.85 \mathrm{mT}$ with the external magnets), the divergence profiles of the two segments look similar to each other, though their intensity is very different (higher for the top one). This decorrelation between intensity and divergence is not clear. It can be addressed to different homogeneity among the beamlet groups inside the beam segments, but could be due also to a problem in the grid system. A grid segment misalignment has been already excluded.

- At higher fields, the bottom beam segment
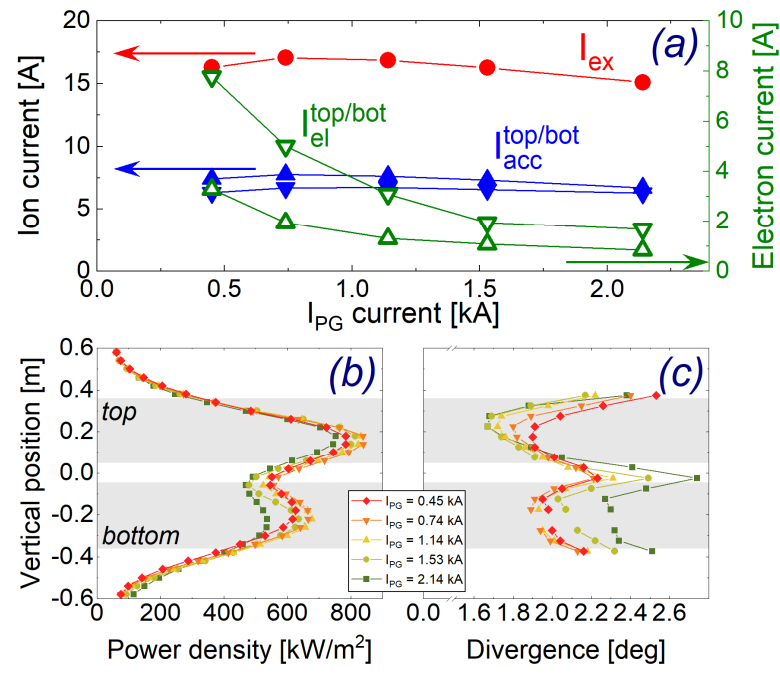

Fig. 3. Dependence of (a) negative ion currents ( $I_{e x}$ and $I_{\text {acc }}$, full symbols) and of co-extracted electrons (empty triangles), of (b) the vertical beam power density from IR calorimetry and of (c) the divergence profile from BES for different values of the filter field ( $I_{P G}$ variation). Parameters: hydrogen, $0.3 \mathrm{~Pa}$, $48 \mathrm{~kW} /$ driver, $\quad j_{e x}=153-168 \mathrm{~A} / \mathrm{m}^{2}, \quad I_{\text {bias }}=100 \mathrm{~A}$, $U_{H V}=7.5 \mathrm{kV}+34.5 \mathrm{kV}, \mathrm{FF}=I_{P G}$ field $+0.4 \mathrm{mT}$.

divergence profile stays flat (almost as flat as before), though increasing the values. The top divergence profile, instead, decreases the minimum monotonically with the field strength.

- In the top segment, the position of the minimum divergence moves upward while increasing the magnetic field, towards the upper limit of the beamlet groups, and the profile becomes more and more peaked. Again, the divergence of the top segment does not follow the direct link between intensity and divergence through the perveance.

- Only the more divergent particles can reach the area between the beam segments [23], explaining why the intensity is lower and the divergence is higher. This two features are emphasized at the higher filter field values.

In conclusion, though the total current carried in the two segments is almost the same, the vertical beam uniformity is highly affected by the intensity of the $I_{P G}$ filter field, which is responsible of the vertical plasma drift in front of the PG. The higher the field, the worse the beam uniformity: already at $I_{P G}=0.45 \mathrm{kA}$ $(\approx 0.85 \mathrm{mT})$, the vertical profile of the beam intensity show a uniformity smaller than $90 \%$. A linear inhomogeneity is suggested in the top beam segment, as from Figure 3 (b); the bottom beam segment looks more uniform but with a worse divergence.

\subsection{Effects of the bias potential}

The biasing of the PG modifies the plasma properties and potentials in front of the apertures, having an impact on the extracted negative ions and then also on the beam 
divergence. In particular, the latter is largely influenced by the PG bias potentials, regardless of the total negative ion extracted current (i.e. regardless of the perveance).

An example of the beam power density changes during a variation in PG biasing at constant RF power is displayed in Figure 4: the control is always in $I_{\text {bias }} ; U_{\text {bias }}$ adjusts accordingly. The color scale of each map is the same, normalized to the maximum power density obtained for the case with $U_{\text {bias }}=16.6 \mathrm{~V}$, to highlight the details of the top beamlet groups. The total extracted negative ion current is decreasing while increasing the bias voltage: $16.6 \mathrm{~A}$ for the case at $16.6 \mathrm{~V}$ becomes $15 \mathrm{~A}$ for the case at $26.1 \mathrm{~V}$. The corresponding total accelerated current follows the same trend as the extracted one and the current density distribution within the beamlet groups changes. Same trends as in the following are observed also in case of constant total extracted negative ion current (not shown here)

The two beam segments look different in the three cases, and have different trends while increasing the bias potential. The decrease in the bottom accelerated current is less pronounced $(-6 \%)$ than for the top $(-13 \%)$, and almost no change in divergence occurs. The almost "frozen" behavior of the bottom segment can be attributed to the presence of the potential rods which seem to act differently in front of the two grid segments. Recent measurements with a movable probe show different plasma potential profiles for the two segments within the beamlet groups in front of the PG [24]. These potential profiles have an impact on the charged particle trajectories in the plasma. In the past, in absence of the potential rods, the bottom beam segment reacted more on bias changing, with similar trends for both the segments (not shown in here); at that time no plasma potential measurements were available.

In the top segment, though the accelerated current is decreasing faster, the divergence is improving (and the global perveance is decreasing). At smaller bias potential, the averaged $2.2 \mathrm{deg}$ of divergence implies the beamlet groups are merging in the horizontal direction; they are more detached at a higher bias potential $(26.1 \mathrm{~V})$ where a lower divergence of $1.9 \mathrm{deg}$ is

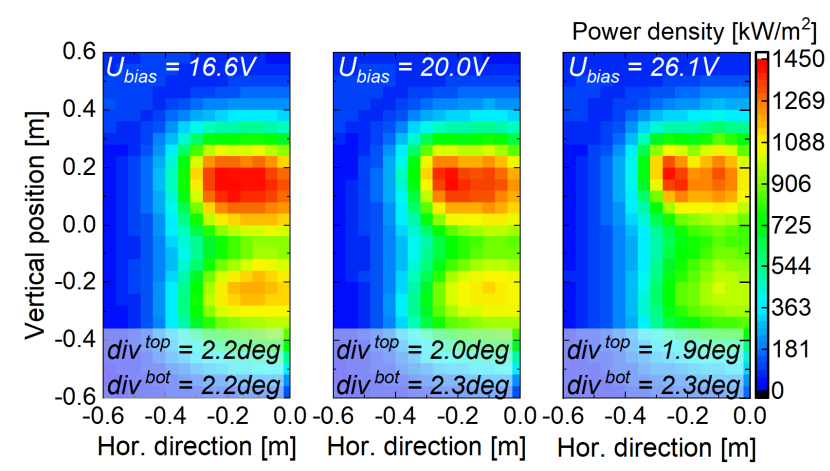

Fig. 4. 2D power density maps at the calorimeter, at different bias voltages; beam divergence, separately for the top and bottom segments, is indicated. Parameters: hydrogen, $\quad 0.3 \mathrm{~Pa}, \quad 48 \mathrm{~kW} /$ driver, $j_{e x}=153-165 \mathrm{~A} / \mathrm{m}^{2}$, $U_{H V}=7.5 \mathrm{kV}+34.5 \mathrm{kV}, \mathrm{FF}=2.3 \mathrm{mT}+0.4 \mathrm{mT}$. achieved.

The bias potential affects the beam divergence regardless of the decrease in the ion currents, $I_{e x}$ and $I_{a c c}$. The difference between the plasma potentials and the bias potential seems to modify the extracted negative ion current density distribution within the beamlet groups inside a beam segment, leading to a change in the beam segment divergence. The divergence changes differently in the two segments, probably according to different plasma sheath properties in front of the $\mathrm{PG}$, which is biased at a constant value for the whole grid.

Asymmetries of plasma potentials in front of the PG and caused by the interplay between plasma generation and filter field are foreseen by fluid codes [25] and PICMCC codes [26], modeling the whole plasma source. Asymmetries in the potentials have been identified also by means of the PIC code ONIX [27] for a single aperture. Global (whole source size) and local (single aperture) phenomena result in asymmetries of the electron and the negative ion distributions within and among beamlet groups. The asymmetry of the extracted negative ion distribution could be responsible of changes in the beamlet (and then beam) divergence, regardless of the total beam current. Only a detailed map of the local plasma potentials within the beamlet groups and for the different segments would be able to clarify the effective role of the potential (and of the plasma drift) on the beam divergence. This has to be accompanied by the knowledge of the extracted current distributions within the beamlet groups and among the different segments. A "local" perveance can be then defined, which becomes then representative (better than the "global" perveance obtained from the total extracted current) of the local beam optics and divergence [28].

\section{Conclusions}

Tuning the beam properties in the ITER NBI to get a uniform low-divergent beam will not be straightforward. Recipes and knobs to tune the beam properties have to be identified in existing ion sources and then transferred to the ITER NBI system. ELISE, with its $1 \mathrm{~m} \times 1 \mathrm{~m}$ ITER-like 3-grid system (though with a limited total acceleration potential, with neither tilted grids nor steering of the beamlets), can provide useful information on the properties of large beams and on how to tune them. Focus of this work is on the vertical beam symmetry (among beam segments) and uniformity (among segments and within beamlet groups) at ELISE. Beam symmetry and uniformity are largely influenced by the interplay of the magnetic filter field and the bias potential (together with the caesium distribution at the PG surface), which are responsible of plasma inhomogeneities in front of the extractor.

The magnetic field created by the current flowing in the PG (as in the ITER NBI) affects the current density within the beam segments, differently for the top and the bottom one. Only by keeping the field strength at very low values (no $I_{P G}$ current, only external magnets $\approx 0.4 \mathrm{mT}$ in the center of the source), a vertical uniform 
beam is obtained, both in intensity and divergence. Minor disuniformities are caused by the Cs distribution determined by the previous conditioning phase. With such a low filter field, the plasma is in an electron-ion regime dominated by electrons, and the negative ions are destroyed by the collisions with energetic electrons.

The experiments here described were done in hydrogen; a direct transfer to deuterium is not straightforward. On one hand, there is the isotope effect to take into account, for which a smaller vertical plasma drift is observed in deuterium with respect to hydrogen for the same source parameters [29]. On the other hand, operation in deuterium requires a much larger strength of the filter field to keep the co-extracted electrons at a tolerable level. In ELISE, at high performances, usually the field strength in case of hydrogen is about 2-3 mT while in deuterium is above $4 \mathrm{mT}$. Due to the high filter field needed in deuterium operation, even in case of a symmetric beam (all the segments carrying the same amount of current), beam uniformity could be poor. A different topology of the magnetic field in combination with or without the $I_{P G}$ current, could solve the conflict between keeping low the amount of co-extracted electrons while preserving a good beam symmetry and homogeneity. Dedicated investigations are ongoing with permanent magnets installed at different positions around the grid system [30].

The bias potential is identified to be a powerful knob to tune the beam divergence at constant perveance. However, the tuning of the divergence works differently for the two beam segments, where plasma potentials and sheath properties could be different because of vertical plasma drifts. The bias potential between the PG and the bias plate, as implemented in ELISE but also in full-size ITER NBI sources, is unique for the whole extraction area. No local tuning of the potential along the vertical dimension is possible, but it would be beneficial at least at the level of beam segments. Detailed investigation of the local plasma sheath properties with a high spatial resolution diagnostic to be compared with single beamlet properties in a large source would be desirable.

Though the investigations on the beam uniformity have been here performed individually for the filter field and the bias potential, their interplay (together with the caesium distribution at the PG surface) increases the complexity of the system, and a simple solution of individual adjustment of the two parameters to get a vertical uniform beam is not straightforward.

\section{Acknowledgments}

The authors would like to thank all of those involved in the operation, maintenance, and support of the ELISE test facility.

This work has been carried out within the framework of the EUROfusion Consortium and has received funding from the Euratom research and training programme 2014-2018 and 2019-2020 under grant agreement No 633053. The views and opinions expressed herein do not necessarily reflect those of the European Commission.

\section{References}

[1] R. Hemsworth et al., Nucl. Fusion 49, 045006 (2009).

[2] R. Hemsworth et al., New J. Phys. 19, 025005 (2017).

[3] G. Serianni and V. Toigo et al., Fusion Eng. Design 146, Part B, 2539-2546 (2019).

[4] V. Toigo et al., New J. Phys. 19, 085004 (2017).

[5] B. Heinemann et al., Fusion Eng. Des. 84, 915 (2009).

[6] R. Hemsworth, A. Tanga, V. Antoni, Rev. Sci. Instrum. 79, 02C109 (2008).

[7] M. Bacal and M. Wada, Applied Phys. Rev. 2, 021305 (2015).

[8] S. Cristofaro et al., Rev. Sci. Instrum. 90, 113504 (2019).

[9] N. den Harder, Private Communication.

[10] C. Wimmer et al., Rev. Sci. Instrum. 91, 013509 (2020).

[11] M. Fröschle, Fus. Eng. Design 88, 1015- 1019 (2013).

[12] D. Wünderlich et al., Plasma Phys Control. Fusion 58, 125005 (2016).

[13] D. Wünderlich et al., AIP Conference Proceedings 2052, 040001 (2018).

[14] R. Nocentini et al., Fusion Eng. Des. 88, 913 - 917 (2013).

[15] I. Mario et al 2020 Nucl. Fusion in press https://doi.org/10.1088/1741-4326/ab8573

[16] A. Hurlbatt, N. den Harder, U. Fantz and NNBI Team, Fus. Eng. Design, 153, 111486 (2020).

[17] J. D. Lawson, Journal of Electronics and Control 5(2), 146-151 (1958).

[18] R. Keller, "The physics and Technology of Ion Sources", chapter 3, page 25, Brown (1989).

[19] C. D. Child, "Discharge from hot CaO", Phys. Rev. (Series I) 32, 492 (1911).

[20] I. Langmuir, The effect of space charge and residual gases on thermionic currents in high vacuum, Phys. Rev. 2, 450 (1913).

[21] F. Bonomo et al., AIP Conference Proceedings 2052, 040002 (2018)

[22] I. Mario et al., "Towards the ITER NBI: impact of the plasma parameters on the performances of a large ITERlike beam", 46 ${ }^{\text {th }}$ European Physical Society Conference on Plasma Physics, Milan (Italy), 8-12 July 2019.

[23] M. Barbisan et al., Plasma Phys. Control. Fusion 59, 055017 (2017)

[24] I. Mario, "Evaluation of plasma and beam properties at the large negative ion source ELISE", Ph.D thesis, University of Augsburg, 2020, to be submitted.

[25] S. Lishev et al., AIP Conference Proceedings 1869, 030042 (2017).

[26] G. Fubiani et al., New J. Phys. 19, 015002. (2017).

[27] I.M. Montellano, "Application of a 3D Monte Carlo PIC code for modeling the particle extraction from negative ion sources", PhD thesis, University of Augsburg, 2019.

[28] N. den Harder et al., Fus. Eng. Design 153, 111507 (2020).

[29] U. Fantz, L. Schiesko, D. Wünderlich and NNBI Team, AIP Conference Proceedings 1515, 187 (2013).

[30] D. Wünderlich et al., Rev. Sci. Instrum. 90, 113304 (2019). 